\title{
Oxidized Phospholipids: Introduction and Biological Significance
}

\author{
Mohammad Z. Ashraf and Swati Srivastava
}

Additional information is available at the end of the chapter

http://dx.doi.org/10.5772/50461

\section{Introduction}

Phospholipids containing polyunsaturated fatty acids are highly prone to modification by reactive oxygen species. They tend to undergo lipid peroxidation to form OxPLs which induce cytotoxicity and apoptosis and plays a significant role in inflammation. There are reports that provide insights for involvement of OxPLs in interleukin transcription, phenotype switching of smooth muscle cells and apoptotic mechanisms of the modified phospholipids. Thus peroxidation greatly alters the physiochemical properties of membrane lipid bilayers and consequently induces signaling depending upon the formation or reorganization of membrane domains or specific molecular binding (Deigner et al, 2008). Distinct OxPLs species may interact with specific binding sites and receptors leading to the activation of individual signaling pathways. The most prevalent human coronary atherosclerosis is a chronic inflammatory disease that occurs due to lipid abnormalities. Pro-inflammatory oxidized low-density lipoprotein (OxLDL) has been suggested to be a link between lipid accumulation and inflammation in vessel walls. Increased levels of phospholipids' oxidation products have been detected in different organs and pathological states, including atherosclerotic vessels (Watson et al 1997, Subbanagounder et al 2000), inflamed lung (Yoshimi et al 2005, Nakamura et al 1998 ), nonalcoholic liver disease (Ikura et al 2006), plasma of patients with coronary artery disease (Tsimikas et al 2005), as well as in apoptotic cells (Huber et al 2002, Chang et al 2004), virus-infected cells (Van Lenten et al 2004) and cells stimulated with inflammatory agonists (Subbanagounder et al 2002). Moreover, studies have been done on two HDL-associated enzymes, serum paraoxonase (PON1) and PAF-acetylhydrolase (PAF-AH), which are responsible for hydrolysis of plasma oxidized phospholipids (Forte et al 2002) thereby providing evidence for their role in atherosclerosis. Another important marker of oxidative stress is the association of OxPLs with the apolipoprotein B-100 particle (OxPLs/apoB) of 
LDL. Increased levels of OxPLs/apoB are implicated in coronary artery disease, progression of carotid and femoral atherosclerosis and the prediction of cardiovascular events (Tsimikas et al 2005).

\section{Formation of OxPLs}

OxPLs are generated by the oxidation of polyunsaturated fatty acid residues, which are usually present in the phospholipids at the $s n-2$ position. Oxidation of phospholipids is initiated either enzymatically by lipoxygenases or by reactive oxygen species and propagates via the classical mechanism of lipid peroxidation chain reaction. This implies that the production of OxPLs cannot be regulated by adjusting the amount or activity of enzymes. Hence there is a probability of the uncontrolled generation of OxPLs during oxidative stress. Several evidences suggest that OxPLs are formed from Poly Unsaturated Fatty Acids (PUFAs) at the sn-2 position (Bochkov et al 2007, Podrez et al 2002). Bioactive oxidized phospholipids may contain fragmentation products of PUFA, such as 1-palmitoyl2-oxovaleroyl-sn-glycero-3-phosphorylcholine and 9-keto-10-dodecendioic acid ester of 2lyso-phosphatidyl choline (KOdiA-PC); prostaglandins, such as 15 deoxy-delta 12, 14 prostaglandin I2 (PGI2) and 1-palmitoyl-2-(5,6-epoxyisoprostane E2)-sn-glycero-3phosphoryl choline (PEIPC); and levuglandins. These molecules exhibit different biological activities. Chromatographic separation of many products formed by oxidation of 1palmitoyl-2-arachidonoyl-sn-glycero-3-phosphorylcholine (PAPC) led to the identification of 1-palmitoyl-2-(5-oxovaleroyl)-sn-glycero-3-phosphatidylcholine (POVPC), 1-palmitoyl-2glutaroyl-sn-glycero-3-phosphatidylcholine (PGPC) and 1-palmitoyl-2-(5,6-epoxyisopropane $\mathrm{E}_{2}$ )-sn-glycero-3-phosphatidylcholine (PEIPC) as potent lipid mediators of inflammation. High structural variation may explain why OxPLs demonstrate a remarkable variety of biological activities (FIGURE-1).

Enzymatic and non-enzymatic reactions, free-radical, and radical-free processes are capable of initiating wide spectrum of reactions causing oxidation of PUFAs. Majority of these reactions produce identical primary oxidation products (i.e., peroxyl radicals and hydroperoxides). Subsequent oxidation of OxPLs is an enzyme-independent stochastic process producing a wide spectrum of OxPLs. Peroxidation products thus generated proceeds according to several mechanisms such as oxidation of PUFA residue, cyclization of peroxyl radical or oxidative fragmentation of esterified PUFAs generating either fulllength residues incorporating several oxygen atoms, or shortened fatty acid residues. Introduction of additional oxygen atoms into PUFAs is a common mechanism that increases complexity of OxPLs mixtures however biological activities of poly-oxygenated PLs are still not characterized. On the other hand, cyclization of peroxyl radical produces cyclic peroxide, which undergoes re-arrangements yielding bicyclic endoperoxide, or oxidation introducing additional non-cyclic or cyclic peroxide group. Cyclization of peroxyl radical is only possible for FAs having three or more double bonds (Salomon et al 2005). 


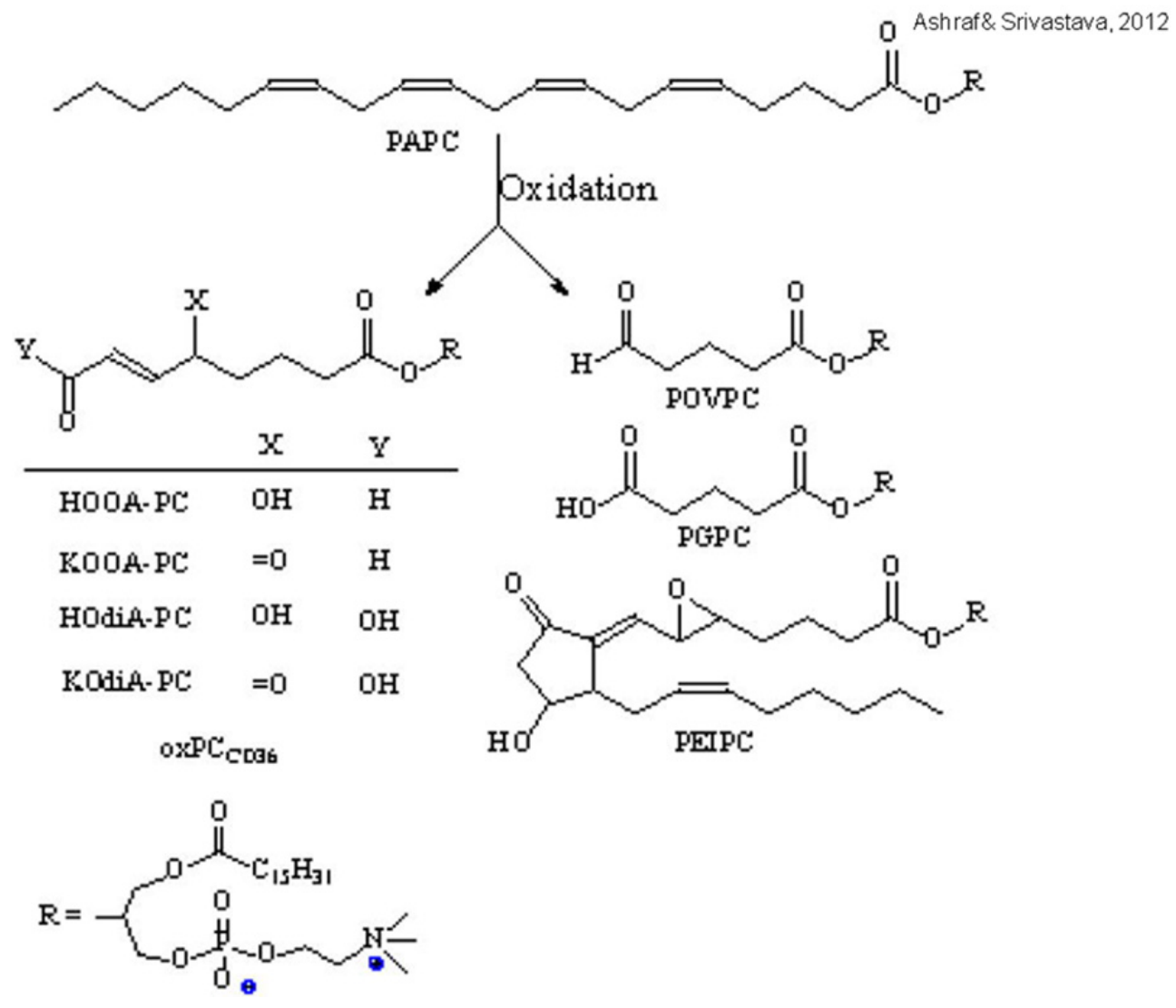

Figure 1. Representative chemical structures of oxidized phospholipids formed during oxidation of PAPC.

\subsection{Oxidative cleavage and generation of fragmented OxPLs species}

Peroxides/ peroxyls are transformed into advanced oxidation products by fragmentation of hydroperoxides. $\gamma$-Hydroxy (or oxo) a,b-unsaturated PLs with terminal aldehyde groups are produced from hydroperoxides via oxidation/fragmentation or polymerization/cleavage. Oxidative fragmentation of hydroperoxides occurs via several mechanisms including bscission, Hock rearrangement, or cyclization of alkoxy radical produced from hydroperoxide (Gugiu et al 2006). $\gamma$-Hydroxy (or oxo)- $\alpha, \beta$-unsaturated aldehyde PLs are highly reactive compounds, that are able to covalently link to amino groups of proteins, as well as thiol groups of biomolecules (Hoff et al 2003). On the other hand, peroxyl radical can cross-react with double bonds present in hydroperoxides yielding peroxydimers, these are unstable products and spontaneously break down forming either new radicals or $\alpha, \beta-$ unsaturated aldehydes (Schneider et al 2008). In addition to these products, saturated fragmented species containing terminal carbonyl groups are produced by oxidative fragmentation of PUFA-PLs, most common amongst which are oxononanoate and azelaoate 
formed from linoleic acid, oxovaleroate, and glutaroate generated from arachidonic acid, or oxobutyrate and succinate produced from docosahexaenoic acid (Gu et al 2003, Podrez et al 2002). Saturated fragmented OxPLs can be formed by further oxidation of $\gamma$-hydroxy (or oxo)- $\alpha, \beta$-unsaturated PLs in addition to direct formation from hydroperoxides, (Podrez et al 2002). Saturated fragmented OxPLs 1 lack double bonds and hence they are resistant to further oxidation as the absence of double bonds within fragmented chains results in reduced reactivity of aldehyde containing saturated OxPLs as compared to $\alpha$, $\beta$-unsaturated fragmented OxPLs.

\subsection{Non-enzymatic oxidation of PL-PUFAs}

This process is initiated by free radicals or non-radical reactive oxygen species (ROS). Free radical-mediated chain reaction is initiated by the formation of carbon-centered radicals and/or hydroperoxides of PUFAs (peroxidation of PUFAs). Due to the presence of methylene groups located between double bonds (bisallylic methylene groups), PUFAs are more susceptible to oxidation as compared to saturated FAs. As a result they are characterized by weakened hydrogen-carbon bonds. Free radicals can abstract hydrogen from bisallylic methylene leading to the formation of carbon-centered radicals within PUFAs. Now occurs the initiation step of lipid peroxidation, Carbon-centered radicals rapidly react with molecular oxygen, producing peroxyl radicals. These Peroxyl radicals react with bisallylic methylene groups in other PUFA molecules, leading to the transformation of peroxyl radicals to hydroperoxides and generation of new carboncentered radicals. Thus, additional cycles of peroxidation are initiated. PUFA hydroperoxides in turn produce reactive alkoxyl and hydroxyl radicals via iron or coppercatalyzed Fenton-like reactions, further propagating the chain reaction (Bochkov et al 2010).

\subsection{Enzymatic oxidation of PL-PUFAs}

1, 4-pentadiene motifs are recognized within unsaturated fatty acids by lipoxygenases (LOXs) and molecular oxygen with high stereoselectivity is introduced. The majority of lipoxygenases oxidize only unesterified PUFAs. Only one group (12/15-LOX) amongst all known LOXs is capable of oxidizing PL-esterified fatty acids. This class of enzymes is present in different biological species and includes mouse, rat, rabbit, bovine, and porcine leukocytetype 12- LOX, rabbit and human reticulocyte-type 15-LOX, and soybean LOX (Huang et al 2008, Wittwer et al 2007). Switching of activity of electron transport in mitochondria to peroxidation by cytochrome c (cyt c) has been suggested by Kagan et al (2005). This transformation begins when cyt $\mathrm{c}$ binds to negatively charged cardiolipin (CL), leading to conformational changes and subsequent release of PL-protein complex from mitochondria into cytosol. The complex of cyt $\mathrm{c}$ with $\mathrm{CL}$ activated by traces of PUFA-OOH or $\mathrm{H}_{2} \mathrm{O}_{2}$ acquires the ability to oxidize CL, PS, or PI, with formation of PL-OOH (Kagan et al 2009).

Alternatively, OxPLs are also generated by re-esterification of free oxidized PUFAs into lyso-PLs. Several types of OxPLs have been found to be generated by this mechanism both in vivo and in vitro (Arai et al 1997, Birkle et al 1984). 


\subsection{Detoxification of reactive OxPLs}

Detoxification of OxPLs comprises the mechanisms that terminate peroxidation chain reaction and inactivate chemically reactive toxic groups produced by oxidation. Hydroxides are characterized by significantly lower chemical reactivity and therefore are considered to be stable and non-toxic compared to hydroperoxides (Spiteller et al 1997). Most commonly, the enzyme catalyzing the reduction of hydroperoxides to hydroxides is glutathione peroxidase (GPx). Lipid hydroperoxides are reduced in a reaction that involve selenocysteine residue of GPx and glutathione thus generating lipid hydroxide and oxidized glutathione. With respect to membrane-bound hydroperoxides of PL esterified PUFAs, PL glutathione peroxidase (GPx4) has the highest activity amongst GPx enzymes (Savaskan et al 2007).

A variety of products containing aldehyde and keto functional groups are formed upon oxidation of OxPLs which are further reduced by aldo-keto reductases to respective hydroxyl groups. Apart from playing physiological role in metabolism of sugar aldehydes, aldo-keto reductases also play a role in detoxification of toxic phospholipid aldehydes (Jin et al 2007).

Another aspect of detoxification is OxPLs cleavage. Platelet activating factor acetylhydrolase (PAF-AH) has been recognized for its ability to cleave and thus inactivate PAF (McIntyre et al 2009).The enzyme was shown to hydrolyze fragmented saturated OxPLs (Stremler et al 1991), as well as long-chain OxPLs, including esterified F2-isoprostanes, PC-hydroperoxides and PEIPC (Kriska et al 2007, Davis et al 2008).

\section{Mechanism of action}

Specific receptor binding of OxPLs is the subject of an ongoing debate. Available evidence suggests that OxPLs interact with various signal transduction receptors and pattern recognition receptors present on the cell surface. Most commonly known receptors include CD36, SRB1, EP2, VEGFR2 and the PAF receptor (Bochkov et al 2007, Zimman et al 2007). It has been demonstrated that when present in vesicles, truncated oxidized fatty acids at the sn-2 position move from the hydrophobic interior to the aqueous exterior of the vesicle. this would allow their recognition by cell surface receptors Earlier models of isoprostanecontaining phospholipids have suggested that they are highly twisted and may distort membrane areas in which they are present (Morrow et al 1992). Moumtzi et al (2007) have shown that phospholipid oxidation products can integrate into lipid membranes of cells and lipoproteins; they can either act as ligands or may cause local membrane disruption. Besides, peroxidation of phospholipids leads to the accumulation of lysoforms as a result of both non-enzymatic decylation and enzymatic hydrolysis reactions catalyzed to a large extent by lipoprotein-as-associated phospholipase $\mathrm{A}_{2}$ (also known as PAF acetylhydrolase), which has high substrate selectivity toward polar phospholipids, including the oxidized forms (Zalewski et al 2005). Some lysophospholipids bind and activate G protein-coupled receptors (GPCR). Parhami et al (1993 \& 1995) explained that oxidized phospholipids act by 
binding to a $G$ protein-coupled receptor. These authors demonstrated that minimally modified LDL stimulated a putative Gs-coupled receptor, thus increasing cyclic AMP (cAMP) levels in endothelial cells. Lysophosphatidylcholine and lysophosphatidic acid triggered the activity of G2A and LPA1-LPA4 receptors respectively (Tomura et al 2005, Anliker et al 2004). In addition to GPCR, OxPLs also activate other classes of receptors such as peroxisome proliferator-activated receptors (PPAR). Thus, phospholipid peroxidation may induce the generation of lysophospholipids that are known to accumulate in LDL (OxLDL) and atherosclerotic lesions (Siess et al 2004, Tselepis et al 2002).

Prostaglandin receptors have been recently implicated into OxPLs-induced inflammation. OXPAPC and its component lipid PEIPC are able to stimulate prostaglandin $\mathrm{E}_{2}$ and $\mathrm{D}_{2}$ receptors (EP2 and DP respectively) and to compete with receptor binding of radio labeled prostaglandin E2 ( $\mathrm{Li}$ et al 2006). Previously, it was observed that POVPC binds to human macrophages via the PAF receptor (PAF-R). Occupancy of the PAF-R by the OxPLs modifies the transcription levels of pro-inflammatory genes such as IL-8 (Pegorier et al 2006).

Some effects of OxPLs are probably not mediated by signal transducing receptors. Modulation of cellular cholesterol depots has been suggested as a non-receptor mediated mechanism of OxPLs sensing by cells. It is well illustrated that OxPAPC induces depletion and re-distribution of cellular cholesterol reserves finally leading to the activation of a transcription factor SREBP, a well recognized sensor for cellular cholesterol contents. In turn, SREBP activates IL-8 production (Yeh et al 2004). The human aortic EC gene expression was found to be stimulated by PAPC. Furthermore, OxPAPC may bind to a 37KDa glycosylphosphatidylinositol anchored protein, which interacts with TLR4 to induce interleukin-8 (IL-8) transcription (Walton et al 2003). Leitinger et al (2003) and Watson et al (1997) have described a possible role of toll-like receptors (TLRs) in OxPLs-induced inflammation. Studies have confirmed that Asp299Gly-TLR4 polymorphism plays a protective role in attenuation of atherosclerosis.

Mitogen activated protein kinase phosphatase-1 (MKP-1) was reported to be involved in OxPAPC-induced MCP-1 production. Also activation of eNOS by OxPAPC is regulated via a phosphatidylinositol-3-kinase/Akt-mediated mechanism, OxPAPC-induced SREBP activation is significantly reduced with eNOS inhibition (Berliner and Gharavi, 2008).

Chen et al (2007) reported that LDL-associated phosphatidylcholine esterified with sn-2azelaic acid at the $s n-2$ position is readily taken up by cells. This compound, one of the main phospholipid oxidation products in LDL, induces apoptosis of HL60 cells at low micromolar concentrations. Since the intact phospholipid is required for signaling, this effect can be prevented by over-expression of PAF acetyl hydrolase known for oxidizing phospholipids with polar residues at the $s n-2$ position.

Another biologically active phospholipid described is platelet activating factor (PAF) having various inflammatory actions such as platelet aggregation, hypotension, anaphylactic shock and increased vascular permeability (Prescott et al 2000). PAF is structurally identified as 10-alkyl-2-acetyl-sn-glycero-3-phosphocholine. Atherogenic effects are also induced by PAF 
by activating monocytes and stimulating smooth muscle cell growth. In contrast to the tightly regulated physiological generation of PAF, uncontrolled processes of free radical oxidation generate analogs of PAF in vivo and in vitro. As a result of this uncontrolled chemical reaction, fragmentation of the residue at sn-2 position occurs and these oxidatively generated PAF mimetics stimulate monocytes, leukocytes and platelets. They are found in atherosclerotic lesions and even in blood from individuals exposed to cigarette smoke (Heery et al 1995).

Other oxidized phospholipids such as POVPC and PGPC have also been shown to play major roles in activation of endothelial cells and induction of leukocyte binding. They are identified as abundant products in oxidized LDL. The effect of POVPC is protein kinase-A dependent leading to the stimulation of the cAMP-mediated pathway (Berliner and Gharavi, 2008).

OxPLs also induces autocrine mediators such as vascular endothelial growth factor (VEGF), which works through activation of transcription factor-4 (ATF4) (Oskolkova et al 2008).

\section{OxPLs receptors}

It has been shown that OxPLs stimulate a number of signal-transducing receptors located on the cell surface or in the nucleus, including $G$ protein-coupled receptors, receptor tyrosine kinases, Toll-like receptors, receptors coupled to endocytosis, and nuclear ligand-activated transcription factors such as PPARs.

\subsection{Prostaglandin receptors}

OxPCs containing esterified PEIPC activate receptors recognizing prostaglandins E2 and D respectively ( $\mathrm{Li}$ et al 2006). Activation of EP2 receptor on ECs results in activation of integrins and increased binding of monocytes.

\subsection{Scavenger receptors}

OxPLs comprise a major group of ligands for scavenger receptors. Different classes of Scavenger receptors range from Class A, B, D, E and F depending upon the nature and type of ligand (FIGURE-2). CD36 have been described as the major receptor expressed on macrophages and involved in the process of atherogenesis and apoptosis. The role of CD36 has been shown to be responsible for recognition of free oxidized phospholipids (Boullier et al 2000, Podrez et al 2000). Also Boullier et al (2000) and Watson et al (1997) have pointed out that oxidized phospholipid is covalently linked to apolipoprotein B-100 in extensively oxidized LDL (e.g. $\mathrm{Cu}^{2+}$-oxLDL) and serve as ligand for CD36. Scavenger receptor- ligand interaction initiates signaling cascades that regulate macrophage activation, lipid metabolism and inflammatory pathways which may influence the development and stability of atherosclerotic plaque. Recent studies have demonstrated the expression of scavenger receptors especially CD36 and SR-BI on platelets suggesting their critical role in platelet hyper-reactivity in dyslipidemia and atheroprogression. 


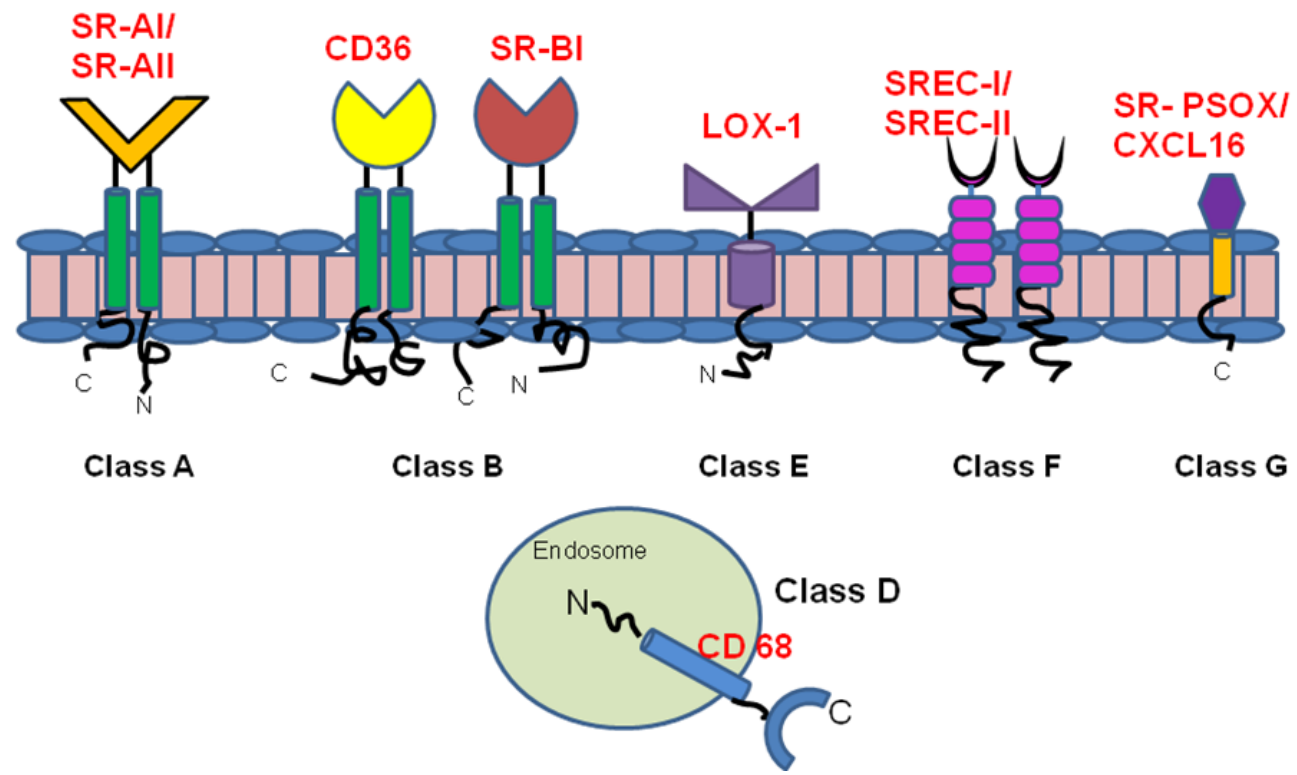

Ashraf \& Srivastava, 2012

Figure 2. Schematic representations of different class of scavenger receptors involved in OxPLs binding.

\subsection{PAF receptors}

OxPLs initiate activation of receptor specific for PAF, which act as an important lipid mediator of inflammation and platelet aggregation. It recognizes alkyl-acylphosphatidylcholines specifically and contains an ether bond at the sn- 1 position in combination with unusually short sn-2 acetyl residue. Oxidative fragmentation of sn-2 PUFAs in alkyl-PCs generates products such as 1-alkyl-2-butenoyl and 1-alkyl-2-butanoyl that are recognized by PAF receptor (Androulakis et al 2005, Marathe et al 1999). However, the role of the PAF receptor in the overall biological activity of OxPCs is not characterized.

\subsection{VEGF receptors}

It has been demonstrated that phosphorylation (activation) of VEGFR2 is enhanced within the first minutes of incubation with OxPAPC (Zimman et al 2007). They hypothesized that trans-activation of VEGFR2 in OxPAPC-treated cells was mediated by c-SRC.

\subsection{Sphingosine-1-phosphate (S1P) receptor 1}

It has shown that OxPAPC stimulates the recruitment of S1P1 to caveolin-enriched membrane microdomains, and induces its phosphorylation (activation) by AKT. Transactivation of S1P1 by OxPAPC plays a role in barrier-protective function of OxPLs. 


\subsection{Toll-like receptor 4}

TLR4 plays a role in OxPAPC-mediated induction of IL-8 in HeLa cells. OxPAPC also induces lung injury and IL-6 production by mouse lung macrophages via the TLR4-TRIFTRAF6 pathway (Imai et al 2008). On the other hand various classes of OxPLs do not influence the basal levels of E-selectin, ICAM-1, VCAM-1, TNFa, IL-6, IL-1a, IL-1b, and COX-2 in whole blood or individual cell types, including human umbilical vein ECs, blood monocytes, macrophage cell line, or fibroblasts (Bochkov et al 2002, Erridge et al 2008).

\subsection{PPAR $\alpha$ and PPAR $\gamma$}

Peroxisome proliferator-activated receptors (PPARs) are intracellular ligand-activated transcription factors. Diacyl-OxPLs stimulated a PPAR response element-driven reporter construct in transfected HAECs and the effect of OxPAPC, POVPC, and PGPC was mediated by PPAR $\alpha$ as indicated by the activation of the ligand binding domain of PPAR $\alpha$, but not PPAR $\gamma$ or PPARS (Lee et al 2000).

Second messengers up-regulated by OxPLs: Apart from the above described receptors, minimally modified Low Density Lipoproteins (MM-LDL) also induces elevation of $\mathrm{Ca}^{2+}$ in ECs (Honda et al 1999) and also OxPAPC was shown to induce rapid and reversible Carpresponses in ECs (Bochkov et al 2002). MM-LDL causes a saturable dose-dependent increase in cAMP levels in aortic ECs that may arise due to activation of Gs and inhibition of Gi heterotrimeric G-protein complexes (Parhami et al 1995).

\section{Biological function}

Many cellular events are initiated and modulated by biologically active oxidized phospholipids. OxPLs were initially characterized as an active principle of minimally modified LDL (MM-LDL), responsible for its ability to stimulate EC to bind the leukocytes (Watson et al 1995). MM-LDL and OxPLs has the characteristic feature of inflammatory agonist i.e., their ability to activate binding of monocytes but not neutrophils (Watson et al 1997). In contrast to lipopolysaccride (LPS), tumor necrosis factor $\alpha$ (TNF $\alpha$ ), or interleukin 1 (IL-1), MM-LDL does not up-regulate the expression of ICAM-1, VCAM-1 and E-Selectin on EC (Kim et al 1994), but promotes surface deposition of CS-1-containing variant of fibronectin (CS-1 FN) serving as ligand for the $\alpha 4 \beta 1$ (VLA-4) integrin expressed on the surface of monocytes (Shih et al 1999). Similar to MM-LDL, OxPLs selectively stimulate adhesion of monocytes by CS-1 FN-dependent mechanism. Likewise other inflammatory agonists, OxPLs also stimulate the production of cyto- and chemokines. OxPLs are known to up-regulate expression IL-6, IL-8, MCP-1, GRO $\alpha$, MIP-1 $\alpha$, MIP-1 $\beta$ and CXCL3 (Subbanagounder et al 2002, Furnkranz et al 2005, Lee et al 2000, Reddy et al 2002, Kadl et al 2002, Gargalovic et al 2006, Huo et al 2001).

Expression of a number of genes related to angiogenesis, atherosclerosis, inflammation and wound healing are modulated by oxidized phospholipids in human aortic endothelial cells (Berliner and Gharavi, 2008; Gargalovic et al., 2006). Bochkov and colleagues (2002, 2007) 
have made known that OxPLs counteract the lipopolysaccride (LPS) pathway. Considering anti-inflammatory role of OxPLs, they reported that oxidized 1-palmitoyl-2arachidonoyl-snglycero-3-phosphocholine (OxPAPC) interfered with the ability of LPS to bind to the LPSbinding protein (LBP) and to CD-14, thus suppressing LPS-induced nuclear factor- $\mathrm{KB}$ (NF$\kappa \mathrm{B})$-mediated up-regulation of inflammatory genes.

Knapp and coworkers (2007) found that OxPAPC inhibits the interaction of LPS with LPSbinding protein and CD14. This also reduces phagocytotic activity of neutrophils and macrophages by a CD-14-independent mechanism. However, in these experiments, administration of OxPAPC rendered mice highly susceptible to Escherichia coli peritonitis, which may cause mortality during gram-negative sepsis in vivo. Thus the overall harmful profile of phospholipid oxidation products includes the impairment of host response to bacterial infections.

Recently, Gharavi and colleagues (2007) have reported the activation of JAK2/STAT3 pathway by phospholipids and implicated their role in atherogenesis. 1-Palmitoyl-2epoxyisoprostane-sn-glycero-3-phosphocholine, an oxidation product of -1palmitoyl-2arachidonoyl-sn-glycero-3-phosphocholine, induces c-Src kinase-dependent activation of JAK2 in endothelial cells and synthesis of chemotactic factors, such as interleukin (IL)-8. In turn, STAT3 activation and regulation of IL-8 transcription is dependent on JAK2 leading to the enhanced levels of STAT3 activity in inflammatory regions of human atherosclerotic lesions. Since STAT3 activation is involved in other chronic inflammatory diseases such as rheumatoid arthritis, psoriasis etc, it has been suggested that STAT3 activation by oxidized phospholipids could be an important interventional target for atherosclerosis and other diseases with inflammatory components.

\subsection{Regulation of vascular cell function}

OxPLs have multiple effects on endothelial cells. After $4 \mathrm{~h}$ treatment with $50 \mu \mathrm{g} / \mathrm{ml}$ of OxPAPC $\sim 1000$ genes are regulated amongst which $\sim 600$ are up-regulated and $\sim 400$ are down-regulated (Gargalovic et al 2006). Also, a major difference in responsiveness to specific effects of Ox-PAPC of endothelial cells from different human donors has been documented (Gargalovic et al 2006). The atherogenic pathways which were found to be upregulated include inflammation, cholesterol synthesis, coagulation and decrease in cell division. Some important effects of OxPAPC on endothelial cell function independent of gene regulation have been reported. OxPAPC has been shown to increase monocytes but not neutrophils binding by activating $\beta-1$ integrin (Berlin et al 2008, Leitinger et al 2005).

Many effects of OxPLs are mediated by its interaction with CD36. Several studies have indicated that LDL supplemented with OxPAPC or vesicles supplemented with fragmented $\alpha / \beta$ unsaturated fatty acids at the sn- 2 position, such as KOdiA or HODA PC, bind to CD36 (Podrez et al 2002, Greenberg et al 2006). Another important phagocytic function of macrophages is the uptake of apoptotic cells, which are abundant in atherosclerotic plaques. OxPLs including oxidized phosphatidyl serine and phosphatidyl choline derivatives were shown to serve as ligands for macrophage uptake of apoptotic cells (Chou et al 2008, Greenberg et al 2006). 
OxPLs also interact and bind with other recognition receptors in macrophages such as TLRs, CD14, LPS binding protein and C-reactive protein competing with negative ligands (Bochkov et al 2007, Bochkov et al 2002, Erridge et al 2008, Miller et al 2003). Thus, the formation of OxPLs during inflammation may represent an important feedback mechanism to limit further tissue damage. OxPLs have also been shown to activate macrophages. Currently conducted studies have revealed the role of OxPAPC in inducing lung injury and cytokine production by lung macrophages (Imai et al 2008).

The role of OxPLs in adaptive immune response can't be overlooked where they modulate the maturation process of dendritic cells (DCs). OxPLs also regulate innate immunity in human leprosy (Cruz et al 2008). In addition to the effects on DCs, OxPLs have also been shown to affect and induce T-cells (Seyerl et al 2008).

Phenotypic switching of smooth muscle cells (SMCs) involving increased proliferation; enhanced migration and down-regulation of SMC differentiation marker genes play a critical role in atherogenesis. Many studies have shown that OxPLs stimulate differentiation and cell division of SMCs (Heery et al 1995, Pidkovka et al 2007) while others have shown activation of apoptotic signaling pathways (Fruhwirth et al 2008).

\subsection{Gene expression}

OxPLs have profound effect on gene expression. OxPAPC have been shown to modulate the expression of approximately 1000 genes in human aortic ECs which include both upregulated and down-regulated mRNAs (Gargalovic et al 2006). OxPLs regulate genes related to inflammation, lipid metabolism, cellular stress, proliferation, and differentiation. These include VEGF-A and IL-8, which are induced by OxPLs independent of their transcription factors.

\subsection{Pathophysiological functions}

Pathophysiologically OxPLs are involved in various proinflammatory and cardiovascular disorder; details are being described below (FIGURE-3).

\subsection{Atherosclerosis}

Quantification of OxPLs using liquid chromatography coupled with mass spectrometry has indicated that atherosclerotic vessels contain high concentrations of OxPCs. Different species of OxPCs were detected in atherosclerotic vessels including PL-hydroperoxides and hydroxides (Waddington et al 2001). In addition to elevated levels of OxPLs, atherosclerotic vessels express high amounts of proteins known to be induced by OxPLs in vitro. The latter includes MKP-1 (Reddy et al 2004), ATF3, ATF4 (Gargalovic et al 2006), SREBP-1 (Yeh et al 2004), HO-1 and IL-8 (Cheng et al 2009), MCP-1 and COX-2 (Ma et al 2008). OxPLs act on all major cell types involved in atherogenesis including monocytes, endothelial and vascular smooth muscle cells, lymphocytes, and platelets. 


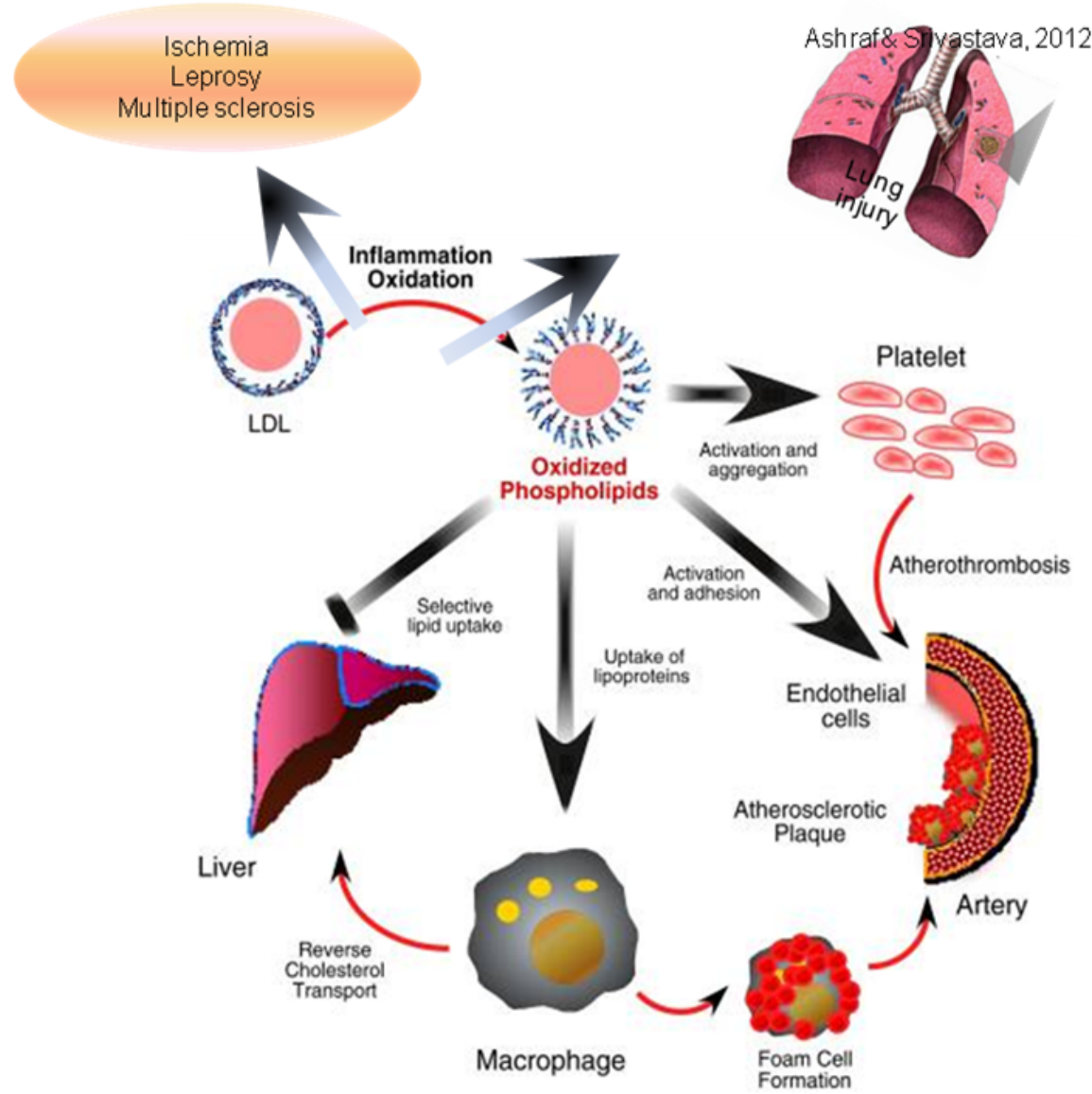

Figure 3. Oxidized phospholipids present in oxidized LDL induce various Diseases.

\subsection{Lung injury}

The epithelial lining pulmonary surfactant is permanently exposed to high concentrations of oxygen and other oxidants present in the air. Ozone gas also plays a role in generating oxidatively truncated PLs (Uhlson et al 2002). Under normal healthy conditions surfactant is protected from oxidation by maintaining low contents of PUFAs, antioxidant action of glutathione present in the lining fluid and surfactant proteins A and D (Kuzmenko et al 2004). However, the accumulation of biologically active OxPLs products occurs in pathological states due to the oxidation of surfactant PCs, membrane lipids and apoptosis of bronchial cells. Studies conducted with animal models have shown that OxPLs protect lungs from acute lung injury. Ma et al. (2004) showed that OxPAPC inhibits elevation of TNFa in mice upon intratracheal or systemic administration of LPS or CpG DNA. Hence the available data shows that OxPLs may induce either beneficial or detrimental effects on lungs. The action of OxPLs on the lungs may depend upon their concentrations, lower levels 
of OxPLs protect endothelial barrier whereas high concentrations of the same OxPLs induce disruptive effects (Birukov et al 2004, DeMaio et al 2006).

\subsection{Ischemia}

Ischemia/reperfusion results in elevated levels of OxPLs both in tissues and systemic levels. PAF like (alkyl-acyl) OxPLs were detected within the first minutes after reperfusion of kidneys after warm ischemia (Lloberas et al 2002). Plasma concentrations of fragmented OxPCs were increased in patients during the reperfusion period after coronary surgery with cardiopulmonary bypass (Frey et al 2000). Hence available data shows that ischemia/reperfusion is a pathological state characterized by elevated local and circulating levels of OxPLs.

\subsection{Inflammation}

Inflammation is characterized by a massive production of ROS. The elevation of circulating levels of OxLDL in response to inflammatory stimuli has already been shown. The OxPLs production in response to inflammation is induced by different cell types including leukocytes. Phorbol ester-stimulated neutrophils and monocytes incubated with PUFA-PCs produced mono- and bishydroperoxides of $\mathrm{PC}$, as well as isoP-PC, thus suggesting that activated phagocytes can oxidize lipids in the surrounding medium (Jerlich et al 2003).

\subsection{Radiation stress}

Formation of OxPLs can be activated by visual and UV-light. OxPLs accumulating in retinas serve as ligands for CD36-dependent phagocytosis of shed photoreceptor outer segments by retinal pigment epithelium; this process is necessary for normal function of the retina (Sun et al 2006). Generation of OxPLs by light exposure has also been shown in skin cells. UVA-1irradiated PAPC containing several OxPLs species induced expression of antioxidant and anti-inflammatory enzyme heme oxygenase- 1 in dermal fibroblasts, keratinocytes, and in a three-dimensional epidermal equivalent model (Gruber et al 2007). Therefore, OxPLs are likely to play a protective role in UVA irradiated skin by inducing HO-1.

\subsection{Leprosy}

Oxidized PCs have been detected in lepromatous (disseminated) leprosy lesions, but not in tuberculous leprosy characterized by stronger host immune response and self-contained infection (Cruz et al 2008). Lepromatous leprosy lesions are characterized by the accumulation of OxPLs, which can counteract innate and specific immune responses, thereby promoting survival.

\subsection{Multiple sclerosis}

Multiple sclerosis (MS) is an autoimmune disease of the brain that causes neurodegeneration. Role of OxPLs in MS is supported by Qin et al. (2007), demonstrating the presence of OxPLs (alone and conjugated to a $15 \mathrm{KDa}$ protein) in extracts of MS lesions 
directly by Western blot analyses using the E06 antibody. OxPLs might be promoting the inflammatory process in MS lesions.

\section{Medical relevance}

Increasing number of studies suggest the role of oxidized phospholipids in development of atherosclerosis by interacting with specific receptors as well as through their reactive groups that can bind covalently to proteins, forming lipid-protein adducts that become dysfunctional. It is a challenge to determine if therapeutic inhibition of the OxPLs interaction with vessel wall cells can inhibit atherosclerosis. Also it will be interesting to identify the lipid oxidation products that activate each response in the various cell types and the receptors or binding molecules and signal transduction pathways activated by these lipids.

Pro-inflammatory oxidized phospholipids are significant predictors of the presence of carotid and femoral atherosclerosis, development of new lesions and increased risk of cardiovascular events (Ashraf et al 2009). Hence oxidized phospholipids could serve as biomarker for diagnosis of coronary artery disease and they could also be used as potential targets for therapeutic intervention.

\section{Conclusions}

The inflammatory profile of OxPLs combines both pro- and anti-inflammatory effects. OxPLs may show detrimental as well as beneficial cellular effects. OxPLs exert pro-inflammatory effects on different cell types such as endothelium where they induce a shift from antithrombotic and anti-inflammatory state to procoagulant and inflammatory phenotype of EC. Although OxPLs stimulate a number of classical inflammation mechanisms, they are not capable of activating many signaling and adhesion events characteristic of acute inflammation, such as activation of the NFkB pathway, expression of ICAM-1 and E-selectin or adhesion of granulocytes. Several studies have provided evidence that OxPLs play an important role in atherosclerosis. In addition, OxPLs also up-regulate monocytes-specific chemokines and stimulate EC to bind monocytes, thus initiating monocytic inflammation. Thus it can be concluded that OxPLs can stimulate and inhibit inflammation depending upon the biological situation. Advancement in this field can be expected from studies that are based on well defined synthetic and labeled OxPLs species and the modern techniques of system biology. Also advances in the knowledge of signaling pathways and the interaction partners of oxidized phospholipid will increase our understanding of inflammatory processes and molecular mechanisms of various diseases such as atherosclerosis. These studies may also help in playing important role in future therapeutic diagnostics.

\section{Abbreviations}

Oxidized phospholipids (OxPL)

Oxidized low-density lipoprotein (OxLDL)

Serum paraoxonase (PON1)

PAF-acetylhydrolase (PAF-AH) 
9-keto-10-dodecendioic acid ester of 2-lyso-phosphatidyl choline (KOdiA-PC)

15 deoxy-delta 12, 14 prostaglandin I2 (PGI2)

1-palmitoyl-2-(5,6-epoxyisoprostane E2)-sn-glycero-3-phosphoryl choline (PEIPC)

Reactive oxygen species (ROS)

Lipoxygenases (LOXs)

Glutathione peroxidase (GPx)

G protein-coupled receptors (GPCR)

Peroxisome proliferator-activated receptors (PPAR)

Toll-like receptors (TLRs)

Vascular endothelial growth factor (VEGF)

Lipopolysaccride (LPS)

Tumor necrosis factor $\alpha(\mathrm{TNF} \alpha)$

Dendritic cells (DCs)

Smooth muscle cells (SMCs)

Multiple sclerosis (MS)

\section{Author details}

Mohammad Z. Ashraf and Swati Srivastava

Genomics Group, Defence Institute of Physiology \& Allied Sciences, India

\section{References}

Androulakis N, Durand H, Ninio E, and Tsoukatos DC. Molecular and mechanistic characterization of platelet activating factor-like bioactivity produced upon LDL oxidation. J Lipid Res 2005; 46: 1923-1932.

Anliker B, Chun J. Cell surface receptors in lysophospholipids signaling. Semin cell Dev Biol 2004;92:1086-1094.

Arai M, Imai H, Metori A, and Nakagawa Y. Preferential esterification of endogenously formed 5-hydroxyeicosatetraenoic acid to phospholipids in activated polymorphonuclear leukocytes. Eur J Biochem 1997; 244: 513-519.

Ashraf M.Z., Kar N. S., Podrez E.A. Oxidized phospholipids: Biomarker for cardiovascular diseases. Int J Biochem Cell Biol 2009; 41: 1241-1244.

Berliner JA and NM Gharavi. Endothelial cell regulation by phospholipid oxidation products. Free Radic Biol Med 2008; 45:119-123.

Birkle DL and Bazan NG. Effect of Kp depolarization on the synthesis of prostaglandins and hydroxyeicosatetra- $(5,8,11,14)$ enoic acids (HETE) in the rat retina. Evidence for esterification of 12 -HETE in lipids. Biochim Biophys Acta 1984; 795: 564-573.

Birukov KG, Bochkov VN, Birukova AA, Kawkitinarong K, Rios A, Leitner A, Verin AD, Bokoch GM, Leitinger N, and Garcia JG. Epoxycyclopentenone-containing oxidized phospholipids restore endothelial barrier function via cdc42 and Rac. Circ Res 2004; 95: 892-901.

Bluml S, Rosc B, Lorincz A, Seyerl M, Kirchberger S, Oskolkova O, Bochkov VN, Majdic O, Ligeti E, and Stockl J. The oxidation state of phospholipids controls the oxidative burst in neutrophil granulocytes. J Immunol 2008; 181: 4347-4353. 
Bochkov VN, Kadl A, Huber J, et al. Protective role of phospholipids oxidation products in endotoxin-induced tissue damage. Nature 2002; 419:77-81.

Bochkov VN, Mechtcheriakova D, Lucerna M, Huber J, Malli R, Graier WF, Hofer E, Binder $\mathrm{BR}$, and Leitinger N. Oxidized phospholipids stimulate tissue factor expression in human endothelial cells via activation of ERK/EGR-1 and Ca(++)/NFAT. Blood 2002; 99: 199-206.

Bochkov VN, Oskolkova OV, Birukov KG, Levonen AL, CJ Binder, Stockl J. Generation and Biological Activities of Oxidized Phospholipids. Antioxidants \& Redox Signaling. 2010; 12: , 1009-59.

Bochkov VN, Philippova M, Oskolkova O, Kadl A, Furnkranz A, Karabeg E, Afonyushkin T, Gruber F, Breuss J, Minchenko A, Mechtcheriakova D, Hohensinner P, Rychli K, Wojta J, Resink T, Erne P, Binder BR, and Leitinger N. Oxidized phospholipids stimulate angiogenesis via autocrine mechanisms, implicating a novel role for lipid oxidation in the evolution of atherosclerotic lesions. Circ Res 2006; 99: 900-908.

Bochkov VN. Inflammatory profile of oxidized phospholipids. Thromb Haemost 2007; 97:348-354.

Boullier A, Gillotte KL, Horkko S, Green SR, Friedman P, Dennis EA et al. The binding of oxidized low density lipoprotein to mouse CD36 is mediated in part by oxidized phospholipids that are associated with both the lipid and protein moieties of the lipoprotein. J Biol chem. 2000; 275:9163-9169.

Byun J, Mueller DM, Fabjan JS, and Heinecke JW. Nitrogen dioxide radical generated by the myeloperoxidasehydrogen peroxide-nitrite system promotes lipid peroxidation of low density lipoprotein. FEBS Lett 1999; 455: 243-246.

Carr AC, Winterbourn CC, and van den Berg JJ. Peroxidasemediated bromination of unsaturated fatty acids to form bromohydrins. Arch Biochem Biophys 1996; 327: 227-233.

Chang MK, Binder CJ, Miller YI, et al. Apoptotic cells with oxidized-specific epitopes are immunogenic and proinflammatory. J Exp Med 2004; 200: 1359-1370.

Chen R, Yang L, MsIntyre TM. Cytotoxic phospholipid oxidation products. Cell death from mitochondrial damage and the intrinsic caspase cascade. J Biol Chem 2007; 282:2484224850.

Cheng C, Noordeloos AM, Jeney V, Soares MP, Moll F, Pasterkamp G, Serruys PW, and Duckers HJ. Heme oxygenase 1 determines atherosclerotic lesion progression into a vulnerable plaque. Circulation 2009; 119: 3017-3027.

Chou MY, K Hartvigsen, LF Hansen, L Fogelstrand, PX Shaw, A Boullier, CJ Binder and JL Witztum. Oxidation-specific epitopes are important targets of innate immunity. J Intern Med 2008; 263: 479-488.

Cruz D, AD Watson, CS Miller, D Montoya, MT Ochoa, PA Seiling, MA Gutierrez, M Navab, ST Reddy, JL Witztum, et al. Host derived oxidized phospholipids and HDL regulate innate immunity in human leprosy. J Clin Invest; 2008; 118:2917-2928.

Davis B, Koster G, Douet LJ, Scigelova M, Woffendin G, Ward JM, Smith A, Humphries J, Burnand KG, Macphee $\mathrm{CH}$, and Postle AD. Electrospray ionization mass spectrometry identifies substrates and products of lipoproteinassociated phospholipase A2 in oxidized human low density lipoprotein. J Biol Chem 2008; 283: 6428-6437. 
DeMaio L, Rouhanizadeh M, Reddy S, Sevanian A, Hwang J, and Hsiai TK. Oxidized phospholipids mediate occluding expression and phosphorylation in vascular endothelial cells. Am J Physiol Heart Circ Physiol 2006; 290: H674-H683.

Erridge C, S Kennedy, CM Spickett and DJ Webb. Oxidized phospholipid inhibition of tolllike receptor (TLR) signaling is restricted to TLR2 and TLR4: roles for CD14, LPSbinding protein and MD2 as targets for specificty of inhibition. J Biol Chem 2008; 283:24748-24759.

Forte TM, Subbanagounder G, Berliner JA, Blanche PJ, Clermont AO, Jia Z et al. Altered activities of anti-atherogenic enzymes LCAT, paraoxonase and platelet activating factor acetylhydrolase in atherosclerosis-susceptible mice. J Lipid Res 2002; 43: 477-485.

Frey B, Haupt R, Alms S, Holzmann G, Konig T, Kern H, Kox W, Rustow B, and Schlame M. Increase in fragmented phosphatidylcholine in blood plasma by oxidative stress. J Lipid Res 41: 1145-1153, 2000.

Fruhwirth GO and a Hermetter. Mediation of apoptosis by oxidized phospholipids. Subcell Biochem 2008; 49:351-367.

Furnkranz A, Schober A, Bochkov VN, et al. Oxidized phospholipids trigger atherogenic inflammation in murine arteries. Arterioscler Thromb Vasc Biol 2005; 25: 633-638.

Gargalovic PS, Gharavi NM, Clark MJ, et al. The unfolded protein response is an important regulator of inflammatory genes in endothelial cells. Arterioscler Thromb Vasc Biol 2006; 26: 2490-2496.

Gargalovic PS, Gharavi NM, Clark MJ, Pagnon J, Yang WP, He A, Truong A, Baruch-Oren T, Berliner JA, Kirchgessner TG, and Lusis AJ. The unfolded protein response is an important regulator of inflammatory genes in endothelial . Arterioscler Thromb Vasc Biol 2006; 26: 2490-2496.

Gargalovic PS, M Imura, B Zhang, NM Gharavi, MJ clark, J Pagnon, WP Yang, A He, A Troung, S Patel et al. Identification of imflammatory gene modules based on variations of human endothelial cell responses to oxidized lipids. Proc Natl Acad Sci USA 2006; 103:12741-12746.

Gharavi NM, Alva JA, Mouillesseaux KP et al. Role of the JAK/STAT pathway in the regulation of interleukin-8 transcription by oxidized phospholipids in vitro and in atherosclerosis in vivo. J Biol Chem 2007; 282: 31460-31468.

Girotti AW and Kriska T. Role of lipid hydroperoxides in photo-oxidative stress signaling. Antioxid Redox Signal 2004; 6: 301-310.

Greenberg ME, M Sun, R Zhang, M Febbraio, R. Silverstein and SL Hazen. Oxidized phosphatidylserine-CD36 interactions play an essential role in macrophage-dependent phagocytosis of apoptotic cells. J Exp Med 2006; 203:2613-2625.

Gruber F, Oskolkova O, Leitner A, Mildner M, Mlitz V, Lengauer B, Kadl A, Mrass P, Kronke G, Binder BR, Bochkov VN, Leitinger N, and Tschachler E. Photooxidation generates biologically active phospholipids that induce heme oxygenase-1 in skin cells. J Biol Chem 2007; 282: 16934-16941.

Gu X, Sun M, Gugiu B, Hazen S, Crabb JW, and Salomon RG. Oxidatively truncated docosahexaenoate phospholipids: Total synthesis, generation, and peptide adduction chemistry. J Org Chem 2003; 68: 3749-3761. 
Gugiu BG, Mesaros CA, Sun M, Gu X, Crabb JW, and Salomon RG. Identification of oxidatively truncated ethanolamine phospholipids in retina and their generation from polyunsaturated phosphatidylethanolamines. Chem Res Toxicol 2006; 19: 262-271.

Heery JM, Kozak M, Stafforini DM, Jones DA, Zimmerman GA, McIntyre TM et al. Oxidatively modified LDL contains phospholipids with platelet-activating factor-like acitivity and stimulates the growth of smooth muscle cells. J clin Invest 1995; 96: 2322-2330.

Hoff HF, O'Neil J, Wu Z, Hoppe G, and Salomon RL. Phospholipid hydroxyalkenals: Biological and chemical properties of specific oxidized lipids present in atherosclerotic lesions. Arterioscler Thromb Vasc Biol 2003; 23: 275-282.

Honda HM, Leitinger N, Frankel M, Goldhaber JI, Natarajan R, Nadler JL, Weiss JN, and Berliner JA. Induction of monocyte binding to endothelial cells by MM-LDL: role of lipoxygenase metabolites. Arterioscler Thromb Vasc Biol 1999; 19: 680-686.

Huang LS, Kang JS, Kim MR, and Sok DE. Oxygenation of arachidonoyl lysophospholipids by lipoxygenases from soybean, porcine leukocyte, or rabbit reticulocyte. J Agric Food Chem 2008; 56: 1224-1232.

Huber J, Valves A, Mitulovic G, et al. Oxidized membrane vesicles and blebs from apoptotic cells contain biologically active oxidized phospholipids that induce monocyteendothelial interactions. Arterioscler Thromb Vasc Biol 2002; 22: 101-107.

Huo Y, Weber C, Forlow SB, et al. The chemokine KC, but not monocyte chemoattractant protein-1, triggers monocyte arrest on early atherosclerotic endothelium. J Clin Invest 2001; 108: 1307-1314.

Ikura Y, Ohsawa M, Suekane T, et al. Localization of oxidized phosphatidylcholine in nonalcoholic fatty liver disease: impact on disease progression. Hepatology 2006; 43: 506-514.

Imai Y, Kuba K, Neely GG, Yaghubian-Malhami R, Perkmann T, van LG, Ermolaeva M, Veldhuizen R, Leung YH, Wang H, Liu H, Sun Y, Pasparakis M, Kopf M, Mech C, Bavari S, Peiris JS, Slutsky AS, Akira S, Hultqvist M, Holmdahl R, Nicholls J, Jiang C, Binder CJ, and Penninger JM. Identification of oxidative stress and Toll-like receptor 4 signaling as a key pathway of acute lung injury. Cell 2008; 133: 235-249.

Jerlich A, Schaur RJ, Pitt AR, and Spickett CM. The formation of phosphatidylcholine oxidation products by stimulated phagocytes. Free Radic Res 2003; 37: 645-653.

Jin $\mathrm{Y}$ and Penning TM. Aldo-keto reductases and bioactivation/detoxication. Annu Rev Pharmacol Toxicol 2007; 47: 263-292.

Kadl A, Huber J, Gruber F, et al. Analysis of inflammatory gene induction by oxidized phospholipids in vivo by quantitative real-time RT-PCR in comparison with effects of LPS. Vascul Pharmacol 2002; 38: 219-27.

Kagan VE, Bayir HA, Belikova NA, Kapralov O, Tyurina YY, Tyurin VA, Jiang J, Stoyanovsky DA, Wipf P, Kochanek PM, Greenberger JS, Pitt B, Shvedova AA, and Borisenko G. Cytochrome c/cardiolipin relations in mitochondria: A kiss of death. Free Radic Biol Med 2009; 46: 1439-1453.

Kagan VE, Tyurin VA, Jiang J, Tyurina YY, Ritov VB, Amoscato AA, Osipov AN, Belikova NA, Kapralov AA, Kini V, Vlasova II, Zhao Q, Zou M, Di P, Svistunenko DA, Kurnikov IV, and Borisenko GG. Cytochrome $\mathrm{c}$ acts as a cardiolipin oxygenase required for release of proapoptotic factors. Nat Chem Biol 2005; 1: 223-232. 
Kim JA, Territo MC, Wayner E, et al. Partial characterization of leukocyte binding molecules on endothelial cells induced by minimally oxidized LDL. Arterioscler Thromb 1994; 14: 427-433.

Knapp S, Matt U, Leitinger N, et al. Oxidized phospholipids inhibit phagocytosis and impair outcome in gram-negative sepsis in vivo. J Immunol 2007; 178: 993-1001.

Kriska T, Marathe GK, Schmidt JC, McIntyre TM, and Girotti AW. Phospholipase action of platelet-activating factor acetylhydrolase, but not paraoxonase-1, on long fatty acyl chain phospholipid hydroperoxides. J Biol Chem 2007; 282: 100-108.

Kuzmenko AI, Wu H, Bridges JP, and McCormack FX. Surfactant lipid peroxidation damages surfactant protein A and inhibits interactions with phospholipid vesicles. J Lipid Res 2004; 45: 1061-1068.

Lee H, Shi W, Tontonoz P, et al. Role for peroxisome proliferator-activated receptor alpha in oxidized phospholipid-induced synthesis of monocyte chemotactic protein-1 and interleukin- 8 by endothelial cells. Circ Res 2000; 87: 516-521.

Leitinger N, Tyner TR, Oslund L, et al. Structurally similar oxidized phospholipids differentially regulate endothelial binding of monocytes and neutrophils. Proc Natl Acad Sci USA 1999; 96: 12010-12015.

Leitinger N. Oxidized phospholipid as triggers of inflammation in atherosclerosis. Mol Nutr Food Res 2005; 49:1063-1071.

Leitinger N. Oxidized phospholipids as modulators of inflammation in atherosclerosis. Curr Opin Lipidol 2003; 14: 421-430.

Li R, Mouillesseaux KP, Montoya D, Cruz D, Gharavi N, Dun M, Koroniak L, and Berliner JA. Identification of prostaglandin E2 receptor subtype 2 as a receptor activated by OxPAPC. Circ Res 2006; 98: 642-650.

Lloberas N, Torras J, Herrero-Fresneda I, Cruzado JM, Riera M, Hurtado I, and Grinyo JM. Postischemic renal oxidative stress induces inflammatory response through PAF and oxidized phospholipids. Prevention by antioxidant treatment. FASEB J 2002; 16: 908-910.

Ma Y, Malbon CC, Williams DL, and Thorngate FE. Altered gene expression in early atherosclerosis is blocked by low level apolipoprotein E. PLoS One2008; 3: e2503.

Ma Z, Li J, Yang L, Mu Y, Xie W, Pitt B, and Li S. Inhibition of LPS- and CpG DNA-induced TN F-alpha response by oxidized phospholipids. Am J Physiol Lung Cell Mol Physiol 2004; 286: L808-L816.

Marathe GK, Davies SS, Harrison KA, Silva AR, Murphy RC, Castro-Faria Neto H, Prescott SM, Zimmerman GA, and McIntyre TM. Inflammatory platelet-activating factorlike phospholipids in oxidized low density lipoproteins are fragmented alkyl phosphatidylcholines. J Biol Chem 1999; 274: 28395-28404.

McEver RP and Cummings RD. Perspectives series: Cell adhesion in vascular biology. Role of PSGL-1 binding to selectins in leukocyte recruitment. J Clin Invest 1997; 100: 485- 491.

McIntyre TM, Prescott SM, and Stafforini DM. The emerging roles of PAF acetylhydrolase. J Lipid Res 2009; 50: S255-S259.

Miller YI, S Viriyakosol, CJ Binder, JR Feramisco, TN Kirkland and JL Witztum. Minimally modified LDL binds to CD14, induces macrophage spreading via TLR4/MD-2 and inhibits phagocytosis of apoptotic cells. J Biol Chem 2003; 278: 1561-1568. 
Morrow JD, JA Awad, HJ Boss, IA Blair and LJ Roberts 2nd. Non-cyclooxygenase-derives prostanoids (F2-isoprostanes) are formed in situ on phospholipids. Proc Natl Acad Sci, USA 1992; 89:10721-10725.

Moumtzi A, M Trenker, K Flicker, E Zenzmaier, R Saf, and A Hermetter. Import and fate of fluorescent analogs of oxidized phospholipids in vascular smooth muscle cells. J Lipid Res 2007; 48:565-582.

Nakamura T, Henson PM, Murphy RC. Occurrence of oxidized metabolites of arachidonic acid esterified to phospholipids in murine lung tissue. Annal Biochem 1998; 262: 23-32.

O’Donnell VB, Eiserich JP, Chumley PH, Jablonsky MJ, Krishna NR, Kirk M, Barnes S, Darley-Usmar VM, and Freeman BA. Nitration of unsaturated fatty acids by nitric oxide-derived reactive nitrogen species peroxynitrite, nitrous acid, nitrogen dioxide, and nitronium ion. Chem Res Toxicol 1999; 12: 83-92.

Oskolkova OV, Afonyushkin T, Leitner A, von Schlieffen E, Gargalovic PS, Lusis AJ, et al. ATF4-dependent transcription is a key mechanism in VEGF up-regulation by oxidized phospholipids: critical role of oxidized $s n-2$ residues in activation of unfolded protein response. Blood 2008; 112: 330-339.

Parhami F, Fang ZT, Fogelman AM, et al. Minimally modified low density lipoproteininduced inflammatory responses in endothelial cells are mediated by cyclic adenosine monophosphate. J Clin Invest 1993; 92: 471-478.

Parhami F, Fang ZT, Yang B, et al. Stimulation of Gs and inhibition of Gi protein functions by minimally oxidized LDL. Arteioscler Thromb Vasc Biol 1995; 15: 2019-2024.

Pegorier S, Stengel D, Durand H, et al. Oxidized phospholipid: POVPC binds to plateletactivating-factor on hman macrophages. Implications in atherosclerosis. Atherosclerosis 2006; 188: 433-443.

Pidkovka NA, OA Cherepanova, T Yoshida, MR Alexander, RA Deaton, JA Thomas, N Leitinger, and GK Owens. Oxidized phospholipids induce phenotypic swithcing of vascular smooth muscle cells in vivo and in vitro Circ Res 2007; 101:792-801.

Podrez E.A, E. Poliakov, Z Shen, R. Zhang, Y. Deng, M Sun, P J Finton, L. Shan, M Febbraio, D P Hajjar et al. A novel family of atherogenic oxidized phospholipids promotes macrophage foam cell formation via the scavenger receptor CD36 and is enriched in atherosclerotic lesions. J Biol Chem 2002; 277:38517-38523.

Podrez EA, Febbraio M, Sheibani N, Schmitt D, Silverstein RL, Hajjar DP, et al. Macrophage scavenger receptor CD36 is the major receptor for LDL modified by monocytegenerated reactive nitrogen species. J Clin Invest 2000; 105: 1095-1108.

Podrez EA, Poliakov E, Shen Z, Zhang R, Deng Y, Sun M, Finton PJ, Shan L, Gugiu B, Fox PL, Hoff HF, Salomon RG, and Hazen SL. Identification of a novel family of oxidized phospholipids that serve as ligands for the macrophage scavenger receptor CD36. J Biol Chem 2002; 277: 38503-38516.

Podrez EA, Poliakov E, Shen Z, Zhang R, Deng Y, Sun M, Finton PJ, Shan L, Gugiu B, Fox PL, Hoff HF, Salomon RG, and Hazen SL. Identification of a novel family of oxidized phospholipids that serve as ligands for the macrophage scavenger receptor CD36. J Biol Chem 2002; 277: 38503-38516.

Prescott SM, Zimmerman GA, Stafforini DM, MsIntyre TM. Platelet-activating factor and related lipid mediators. Annu Rev Biochem 2000; 69: 419-445. 
Qin J, Goswami R, Balabanov R, and Dawson G. Oxidized phosphatidylcholine is a marker for neuroinflammation in multiple sclerosis brain. J Neurosci Res 2007; 85: 977-984.

Reddy ST, Grijalva V, Ng C, et al. Identification of genes induced by oxidized phospholipids in human aortic endothelial cells. Vascul Pharmacol 2002; 38: 211-218.

Reddy ST, Nguyen JT, Grijalva V, Hough G, Hama S, Navab M, and Fogelman AM. Potential role for mitogenactivated protein kinase phosphatase-1 in the development of atherosclerotic lesions in mouse models. Arterioscler Thromb Vasc Biol 2004; 24: 1676-1681.

Salomon RG. Levuglandins and isolevuglandins: Stealthy toxins of oxidative injury. Antioxid Redox Signal 2005; 7: 185-201.

Savaskan NE, Ufer C, Kuhn H, and Borchert A. Molecular biology of glutathione peroxidase 4: From genomic structure to developmental expression and neural function. Biol Chem 388: 1007-1017, 2007.

Schneider C, Porter NA, and Brash AR. Routes to 4- hydroxynonenal: fundamental issues in the mechanisms of lipid peroxidation. J Biol Chem 2008; 283: 15539-15543.

Seiss W, Tigyi G. Thrombogenic and artherogenic activities of lysophosphatidic acid. J Cell Biochem 2004; 92: 1086-1094.

Seyerl M, S Bluml, S. Kirchberger, VN Bochkov, O Oskolkova, O Majdic and J stockl. Oxidized phospholipid induce enery in human peripheral T cells. Eur J Immunol 2008; 38:778-787.

Shih PT, Elices MJ, Fang ZT, et al. Minimally modified low-density lipoprotein induces monocyte adhesion to endothelial connecting segment- 1 by activating beta1 integrin. J Clin Invest 1999; 103: 613-625.

Spiteller P and Spiteller G. 9-Hydroxy-10,12-octadecadienoic acid (9-HODE) and 13hydroxy-9,11-octadecadienoic acid (13-HODE): Excellent markers for lipid peroxidation. Chemistry and Physics of Lipids 1997; 89: 131-139.

Stremler KE, Stafforini DM, Prescott SM, and McIntyre TM. Human plasma plateletactivating factor acetylhydrolase. Oxidatively fragmented phospholipids as substrates. J Biol Chem 1991; 266: 11095-11103.

Subbanagounder G, Deng Y, Borromeo C, et al. Hydroxy alkenal phospholipids regulate inflammatory functions of endothelial cells. Vascul Pharmacol 2002; 38: 201-209.

Subbanagounder G, Leitinger N, Schwenke DC et al. Determinants of bioactivity of oxidized phospholipids. Specific oxidized fatty acyl groups at the sn-2 position. Arterioscler Thromb Vasc Biol 2000; 20: 2248-2254.

Subbanagounder G, Wong JW, Lee H, et al. Epoxyisoprostane and epoxycyclopentenone phospholipids regulate monocyte chemotactic protein- 1 and interleukin- 8 synthesis. Formation of these oxidized phospholipids in response to interleukin-1 beta. J Biol Chem 2002; 277: 7271-7281.

Sun M, Finnemann SC, Febbraio M, Shan L, Annangudi SP, Podrez EA, Hoppe G, Darrow R, Organisciak DT, Salomon RG, Silverstein RL, and Hazen SL. Light- induced oxidation of photoreceptor outer segment phospholipids generates ligands for CD36-mediated phagocytosis by retinal pigment epithelium: A potential mechanism for modulating outer segment phagocytosis under oxidant stress conditions. J Biol Chem 2006; 281: 4222-4230.

Tomura H, Mogi C, Sato K et al. proton-sensing and lysolipid-sensitive G-protein coupled receptors: a novel type of multi-functional receptors. Cell signal 2005; 17: 1466-1467. 
Tselepis AD, John Chapman M, Inflammation, bioactive lipids and atherosclerosis: potential roles of a lipoprotein-associated phospholipase A2, platelet activating factoracetylhydrolase. Atheroscler Suppl 2002; 3: 57-68.

Tsimikas S, Brilakis ES, Miller ER, et al. Oxidized phospholipid, Lp(a) lipoprotein, and coronary artery disease. N Engl J Med 2005; 353: 46-57.

Uhlson C, Harrison K, Allen CB, Ahmad S, White CW, and Murphy RC. Oxidized phospholipids derived from ozonetreated lung surfactant extract reduce macrophage and epithelial cell viability. Chem Res Toxicol 2002; 15: 896-906.

Van Lenten BJ, Wagner AC, Navab M, et al. D-4F, an apolipoprotein A-1 mimetic peptide, inhibits the inflammatory response induced by influenza A infection of human type II pneumocytes. Circulation 2004; 110:3252-3258.

Waddington E, Sienuarine K, Puddey I, and Croft K. Identification and quantitation of unique fatty acid oxidation products in human atherosclerotic plaque using highperformance liquid chromatography. Anal Biochem 2001;292: 234-244.

Walton KA, Hsieh X, Gharavi N, Wang S, Wang G, Yeh M, Cole AL, and Berliner JA. Receptors involved in the oxidized 1-palmitoyl 2-arachidonoyl-sn-glycero-3phosphorylcholine-mediated synthesis of interleukin-8. A role for Toll-like receptor 4 and a glycosylphosphatidylinositol anchored protein. J Biol Chem 2003; 278: 29661-29666.

Watson AD, Berliner JA, Hama SY, et al. Protective effect of high density lipoprotein associated paraoxonase. Inhibition of the biological activity of minimally oxidized low density lipoprotein. J Clin Invest 1995; 96: 2882-2891.

Watson AD, Leitinger N, Navab M et al. Structural identification by mass spectrometry of oxidized phospholipids in minimally oxidized low density lipoprotein that induced monocyte/endothelial interactions and evidence for their presence in vivo. J Biol chem 1997; 272: 13597-13607.

Winterbourn CC, van den Berg JJ, Roitman E, and Kuypers FA. Chlorohydrin formation from unsaturated fatty acids reacted with hypochlorous acid. Arch Biochem Biophys 1992; 296: 547-555.

Wittwer J and Hersberger M. The two faces of the 15- lipoxygenase in atherosclerosis. Prostaglandins Leukot Essent Fatty Acids 2007;77: 67-77.

Yeh M, Cole AL, Choi J, Liu Y, Tulchinsky D, Qiao JH, Fishbein MC, Dooley AN, Hovnanian T, Mouilleseaux K, Vora DK, Yang WP, Gargalovic P, Kirchgessner T, Shyy JY, and Berliner JA. Role for sterol regulatory element-binding protein in activation of endothelial cells by phospholipid oxidation products. Circ Res 2004; 95: 780-788.

Yoshimi N, Ikura Y, Sugama Y et al. Oxidized phosphatidylcholine in alveolar macrophages in idiopathic interstitial pneumonias. Lung 2005; 183: 109-121.

Zalewski A, Macphee C. Role of lipoprotein-associated phospholipase A2 in atherosclerosis: biology, epidemiology and possible therapeutic target. Arterioscler Thromb Vasc Biol 2005; 25: 923-931.

Zimman A, K P Mouillesseaux, T Le, N M Gharavi, A Ryvkin, T G Graeber, T T Chen, A D Watson and J A Berliner. Vascular endothelial growth factor receptor 2 plays a role in the activation of aortic endothelial cells by oxidized phospholipids. Arterioscler Thromb Vasc Biol 2007; 27: 332-338. 